\title{
Clinical Study Exploring the Causes of Neck Pain and Disability as Perceived by Those Who Experience the Condition: A Mixed-Methods Study
}

\author{
David M. Walton, Bradley Balsor, and Evelyn Etruw \\ School of Physical Therapy, Western University, 1201 Western Road, London, ON, Canada N6G 1A1 \\ Correspondence should be addressed to David M. Walton, dwalton5@uwo.ca
}

Received 21 August 2012; Accepted 14 October 2012

Academic Editors: M. P. Galea and S. May

Copyright (๑) 2012 David M. Walton et al. This is an open access article distributed under the Creative Commons Attribution License, which permits unrestricted use, distribution, and reproduction in any medium, provided the original work is properly cited.

\begin{abstract}
Designing effective treatment protocols for neck-related disability has proven difficult. Disability has been examined from structural, emotional, and cognitive perspectives, with evidence supporting a multidimensional nature. The patient's perspective of their condition has found increasing value for patient-centred, evidence-informed care. This cross-sectional study utilized descriptive thematic analysis to examine perceptions of causation in 118 people with neck pain. The Brief Illness Perceptions Questionnaire was used to capture perceptions of causation for neck pain symptoms. The Neck Disability Index, the Pain Catastrophizing Scale, the Hospital Anxiety and Depression Scale, and the P4 pain intensity numeric rating scale were also collected. Eight main themes were found for the cause(s) of neck pain: posture and movement, structure and mechanism, emotions, predisposition and lifestyle, symptoms, fatigue and insomnia, treatment, and environment. A series of regression models stratified by perceived cause suggested that disability could be explained by different constructs across the larger of the main themes. The findings are discussed in terms of the false view that mechanical neck pain should be considered a homogenous condition and potential application to treatment decision making based on patient perspectives.
\end{abstract}

\section{Introduction}

Neck pain is a common occurrence in the general population, with a 1 -year incidence of $11-18 \%[1,2]$. The intensity of symptoms can range from mild to extreme, with interference ranging from low to very high. The Neck Pain Task Force [3] has proposed a classification system for neck pain that incorporates both the intensity and resultant interference of neck pain to be used as a treatment decision aide. This classification system encourages separate consideration of symptom intensity and symptom interference, recognizing the two are not always directly linked.

The occasionally ambiguous relationship between pain intensity and disability leads to difficulty in treatment decisions. The mechanisms of neck pain and disability are clearly not well understood. Disability, in particular, has been examined from a variety of perspectives, including structural $[4,5]$, psychological $[6-8]$, and social [9-11] determinants. One of the primary barriers to develop effective treatment paradigms for neck-related disability is the weak, or at best, inconsistent association often seen between findings of structural pathology on diagnostic imaging and presenting signs or symptoms [12]. It has also been suggested that cognitive or emotional processes play a strong role in the genesis of neck-related disability, such as depression and anxiety [13] or pain catastrophizing [14] which may intervene in the relationship between structural pathology and disability. Given that no one mechanism appears to explain all variance in self-reported disability, it is clear that neck-related disability is not a unidimensional construct but must be appreciated from a variety of perspectives across different disciplines. Not the least of which is the patient's own perspective of their condition.

One attractive model for understanding the experience of pain and disability, and the stimuli for seeking treatment, is Leventhal's Common Sense Model of Illness Representations [15]. According to this model, one constructs an understanding of his/her illness or condition through a decision-making and comparative process that incorporates beliefs about the cause, intensity, duration, potential adverse 
effects of the condition and efficacy of available treatment. Dependent on the outcome of that process, a person may or may not choose to modify their behavior, or seek medical intervention. Understanding a patient's illness representation may have impact on clinical outcomes. Phillips et al. [16] have provided evidence of a positive association between a physician's use of the illness-representations approach to intervention and the likelihood of patient compliance with treatment recommendations.

Backed by this evidence and clinical experience, we believe that understanding the sufferer's perception of their condition, including its cause(s) can lend valuable insight into the best approach to individual treatment. The Illness Representations model provides a platform from which to start exploring these constructs. The Brief Illness Perceptions Questionnaire (BIPQ) [17] has been constructed as a shortened version of the full IPQ [18] to allow illness representations to be captured in a relatively simple manner. It is composed of 9 numeric rating-type scales and an openended question on causation. The causation question asks the respondent to list in order of importance the top 3 things they believe are causing their symptoms. For the purposes of this study, we were primarily interested in understanding how people with neck pain understand the cause(s) of their symptoms. Qualitative analysis can be a useful tool at this stage, where the goal is to develop greater understanding in an area that is currently largely unexplored. This paper describes a thematic analysis of the responses to that final question on causation, and then introduces quantitative exploration into the different influences on neck-related disability when stratified by causal belief pattern.

\section{Methods}

Subjects for this study were recruited from a network of outpatient physiotherapy treatment clinics across Canada from November 2010 to August 2012. Subjects were eligible if they were experiencing neck pain or stiffness, defined as occurring within the region bound by the occiput superiorly and the 2 nd thoracic vertebra inferiorly, and laterally to the bilateral ends of the scapular spines (by body diagram). Subjects were not excluded if they described additional symptoms outside of this area, but were excluded if the cause of their symptoms was related to bony pathology (i.e., fracture or dislocation), cancer, lung disease, or other systemic illness. All subjects were between the ages of 18 and 65 and could read and understand English at a conversational level. Approval for this study was obtained from the Health Science Research Ethics Board at Western University Canada.

The design was a cross-sectional, single session data collection protocol. Subjects completed a demographic questionnaire, the Brief Illness Perceptions Questionnaire (BIPQ), the Neck Disability Index (NDI) [19], the Pain Catastrophizing Scale (PCS) [20], the Hospital Anxiety and Depression Scale [21] that includes depression $\left(\mathrm{HADS}_{\text {dep }}\right)$ and anxiety (HADS anx $)$ subscales, the Tampa Scale for Kinesiophobia (TSK) as a measure of fear of movement/(re)injury [22], and the $\mathrm{P} 4$ pain intensity numeric rating scale [23]. The causation question on the BIPQ specifically asked "Please list in rank order up to the 3 most important factors you believe are causing your neck symptoms." The length of answers was not restricted, but point form was encouraged. The P4 is a collection of $4,0-10$ pain intensity scales that asks respondents to indicate their pain intensity in the morning, afternoon, evening and with activity. It has shown superior responsiveness compared to single-item numeric rating scales [23]. The NDI is the most commonly used self-reported neck disability scale currently available [24], and has demonstrated good psychometric properties [25]. It includes 10 domains that are each scored from 0 to 5 , for an overall scale range of 0 to 50 where 0 indicates no disability and 50 indicates complete disability. Both the TSK and PCS have been Rasch analyzed by our group and have demonstrated good to excellent congruence with axioms of quantitative measurement in neck pain $[26,27]$. The HADS has been used extensively in research on people with mechanical neck pain [28-31].

Subjects also underwent a brief clinical exam to collect additional predictive variables for planned regression analyses beyond those that are strictly self-reported. Three variables were collected: the total number (out of 6) of restricted planes of cervical movement (CROM), pressure pain threshold (PPT) bilaterally over the angle of the upper trapezii (UFT), and bilaterally over the belly of the tibialis anterior (TA) muscles. The clinical data were captured by experienced orthopedic clinicians, all of whom underwent a training protocol in order to conduct the test procedures correctly. A pilot study revealed that the CROM protocol, in which each cervical movement (flexion, extension, left and right sideflexion, left and right rotation) was rated as full (coded 0) or restricted (coded 1) demonstrated good interrater reliability $\left(\mathrm{ICC}_{2,1}\right.$ 0.71, unpublished). The PPT protocol has been developed and tested extensively by our group and has shown adequate clinimetric properties of interrater and test-retest reliability [32]. The value recorded was the mean of 3 measures taken bilaterally at each site, measured in $\mathrm{kPa}$.

2.1. Analysis. Sample characteristics were summarized descriptively (frequencies, means, and standard deviations). All responses to the final open-ended question were extracted from the BIPQ and collated in a large, anonymized database. Two researchers, one experienced in qualitative methods and one clinician-scientist, independently performed a descriptive analysis on the items by grouping items into themes. No a priori decisions regarding the number or names of themes were conducted, rather the themes emerged through the analysis in keeping with traditional descriptive methodology [33]. Checking and rechecking of each item and theme were conducted to ensure that the final themes were exhaustive while not redundant. Once first-pass themes were identified, the second pass analysis involved identifying themes that could be logically grouped to form a metatheme, with each subtheme defining conceptually similar elements. The two researchers and a third independent researcher who was not involved in the first or second-pass analyses came 
TABLE 1: Characteristics of the sample.

\begin{tabular}{lc}
\hline$N=118$ & \\
\hline Sex (\% female) & $79 \%$ \\
Age (mean, range) & 47 years $(18$ to 65$)$ \\
Chronic symptoms (>6 months) & $63.6 \%$ \\
Cause & \\
$\quad$ Traumatic & $51.1 \%$ \\
$\quad$ Insidious & $33.9 \%$ \\
$\quad$ Unknown/other & $16.1 \%$ \\
P4 (mean/10, range) & $5.3(0.5$ to 9.5$)$ \\
NDI (mean/50, range) & $17(3$ to 44$)$ \\
\hline
\end{tabular}

together to discuss the themes and to address inconsistencies. Any disagreements were settled through consensus of all 3 researchers. The metathemes were named and representative descriptions were pulled from the pool of items to describe each. The themes were then coded, and each subject in the original database was assigned up to 3 theme codes based on their responses to the causation question. In some cases a subject received only 1 code, which would occur when all 3 of their perceived causes fell into the same theme. In other cases a subject received 2 or 3 codes, when their perceived causes fell into 2 or 3 different themes, respectively.

A series of backwards multiple linear regression models were then built for subjects in each of the theme classifications. Due to the unpredictable nature of the thematic analysis, we could not plan a priori analyses, so these were considered exploratory rather than confirmatory. Any theme for which at least 30 subjects were classified was included in this analysis. The dependent variable was NDI score, and, for each theme classification, the following predictor variables were entered: P4, PCS, TSK, HADS ${ }_{\text {dep }}$, HADS ${ }_{\text {anx }}$, CROM, UFT PPT, and TA PPT. A variable was retained when removal from the model resulted in change in $F$ that was significant at the $P<0.05$ level. To avoid spurious results, assumptions of regression were evaluated prior to and after the models were built: nonmulticollinearity, normality, and homoscedasticity. The former 2 were evaluated through a correlation matrix and evaluation of skewness and kurtosis statistics, respectively. The latter was evaluated through inspection of a plot of predicted to residual estimates. To determine the strongest contributors to NDI score by thematic class, we evaluated standardized beta coefficients and $r^{2}$, reported as percent variance explained, for each variable in the final regression model.

\section{Results}

118 subjects from 10 clinics provided 321 perceived causes of their symptoms (mean 2.7 causes per respondent). The characteristics of the sample are described in Table 1. The sample appeared to be representative of the overall population of people with neck pain, being $79 \%$ female with a mean pain intensity rating of 5.3/10. The two independent researchers thematically analyzed all 321 described causes. After checking, rechecking, discussion with a third

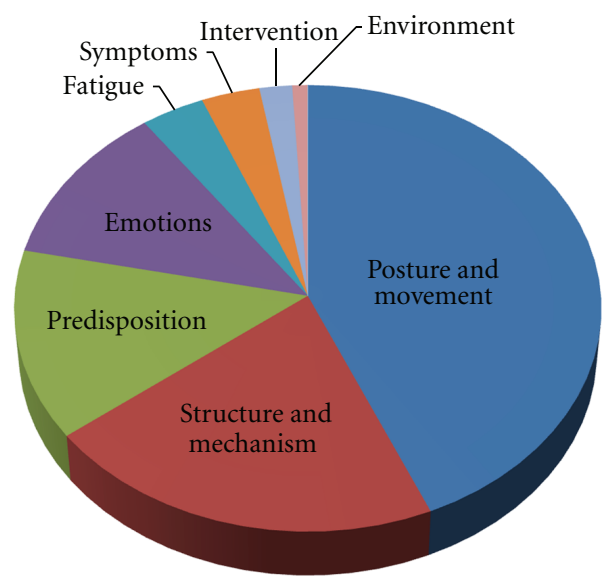

Figure 1: Pie chart depicting the 8 themes identified from the descriptive analysis of 321 perceived causes of neck pain.

researcher, and consensus, 8 metathemes were identified that adequately described all of the data (Figure 1). The themes are described below in decreasing order of proportion of overall responses.

3.1. Theme 1: Posture and Movement (43.5\%). The most commonly-described trigger of neck pain symptoms was broadly classified into postures or movements. The term "posture" was often associated with the terms "static" or "sustained" and was the single most common cause described of the entire database $(n=73,22.7 \%$ of entire database). Respondents described a variety of static postures as triggers, including driving, reading, sleeping, using a computer, sitting, and standing.

Movements were also frequently described but less often than sustained postures. These included heavy lifting $(n=8)$, repetitive movement $(n=6)$, rotation or sudden movement $(n=5)$, and reaching forward or overhead $(n=3)$. Some respondents identified recreation or physical activity as a trigger, with two specific examples (yoga, bowling) offered.

Work postures and activities were grouped separately for the purposes of this analysis as we held the opinion that those who specifically identified work as a trigger of their symptoms may differ in their perceptions of disability from those who did not. Of our sample, $6.9 \%$ of respondents specifically identified work postures or movements as a trigger for their symptoms. Descriptors included "office work," "job: marking and reading," "work desk and chair not properly aligned," or "not having breaks at work to allow stretching."

3.2. Theme 2: Mechanism, Structures, and Tissues (21.1\%). Of the entire database, $14.6 \%$ of respondents identified dysfunction with general structures (e.g., "stiffness in the neck") or specific tissues (e.g., "bad discs") as the cause of their symptoms, while $6.5 \%$ described the mechanism of injury as the cause without relating it to a specific tissue. Of all themes identified, this was the most biomedical in nature. The most common tissues identified were the muscles of 
TABLE 2: Results of multiple linear regression analysis by subtheme.

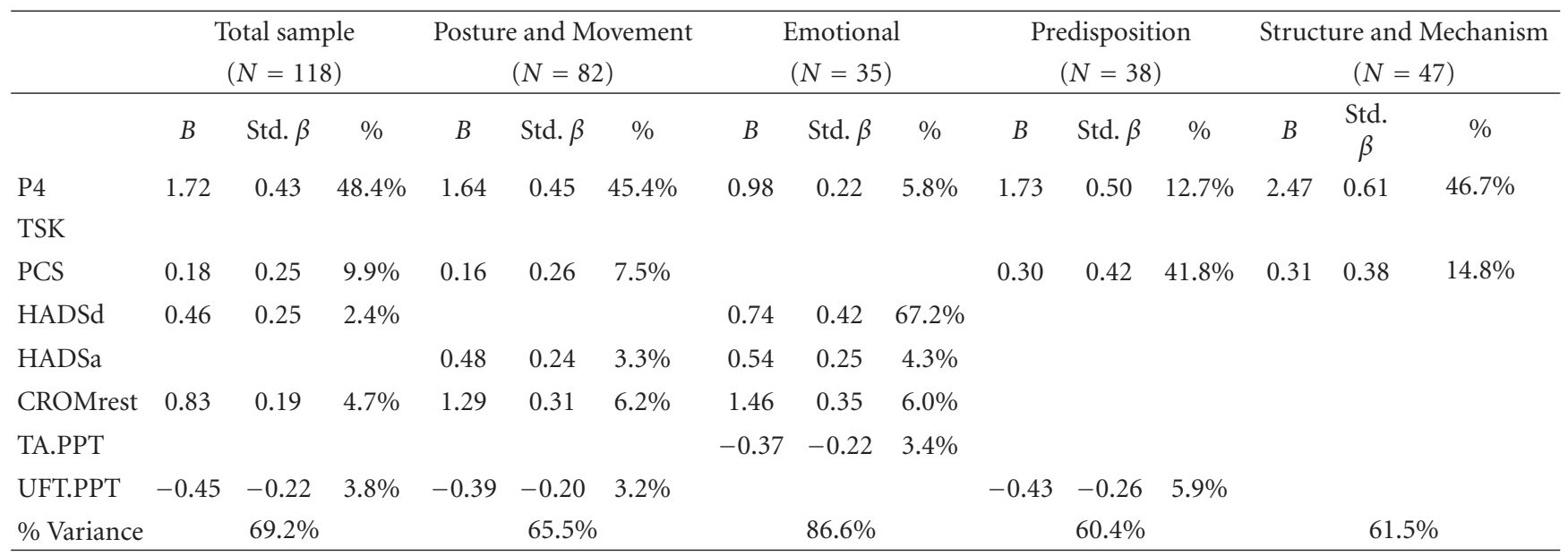

$B$ : unstandardized beta; std. $\beta$ : standardized beta; \%: percent variance explained. Dependent variable is Neck Disability Index for all analyses.

the neck (e.g., "muscle damage," "muscle strain," "muscles are tight"), followed by joint stiffness, disc problems, nerve damage, and ligament damage, in that order. One respondent specifically identified inflammation while another described the rotator cuff of the shoulder as the cause of their neck symptoms. The most common mechanism described $(n=$ 10) was a motor vehicle accident, followed by a collection of other traumas such as falling in a store, a dog accident, a strain during surgery, or a concussion.

3.3. Theme 3: Predisposition or Lifestyle Factors (13.7\%). Responses that formed this theme were related to the belief that some people were vulnerable to neck symptoms by virtue of some preexisting condition or lifestyle behaviour. Subthemes here included improper or too little exercise, overuse or heavy sports activity, genetic predisposition, arthritis or other comorbidity, age, and weight. Weakness or lack of exercise was the most common sub-theme $(n=22)$.

3.4. Theme 4: Emotional Triggers (11.8\%). Some respondents recognized an emotional trigger as the cause of their symptoms, with stress being by far the most common subtheme $(n=23)$. Other emotional constructs included anxiety $(n=3)$, depression $(n=2)$, and a number of one-off descriptors such as denial, doubt, and family troubles.

3.5. Theme 5: Fatigue or Insomnia (3.7\%). This theme was described by two easily recognizable subthemes: fatigue and lack of sleep. Based on the responses, these subjects felt that fatigue or lack of sleep was causing their neck symptoms.

3.6. Theme 6: Symptom Factors (3.4\%). This theme was difficult to interpret and may have been a result of respondents' misunderstanding of the task. The question as posed asked respondents to indicate the causes of their symptoms, but on 11 occasions, the symptoms themselves were described.
These included pain, numbness in the arms or legs, and headaches.

3.7. Theme 7: Intervention-Related Factors (1.9\%). Relatively few respondents indicated that factors related to the treatment they were receiving were causes of their symptoms. However, rather than being due to the active treatment itself, the descriptors in this theme suggested that it was a lack of treatment, or perhaps more appropriately, a lack of knowledge, that was the cause of their symptoms. Descriptors here included "not enough treatment," "not enough assistance or training in what I need to do to help fix it," "not following up right away when aggravated," and "knowledge on how far to push myself." This suggested that these respondents felt an important component of treatment was missing from their regimen. The causal link is not entirely clear here, but may be a result of a sense of distress at not understanding their condition or its triggers.

3.8. Theme 8: Environmental Triggers (0.9\%). Three respondents identified an environmental trigger. Two indicated that the weather was a cause of their symptoms, while another blamed an uncomfortable mattress.

3.9. Evaluating Influences on Disability. Given the distribution of results, we were able to conduct a series of exploratory backward multiple linear regression analyses for the following groups: (1) the entire sample $(N=118)$, (2) those who reported at least one posture and movementrelated cause $(N=82)$, (3) those who reported at least one emotional cause $(N=35)$, (4) those who reported at least one predisposition or lifestyle-related cause $(N=$ 38 ), and (5) those who reported at least one structure or mechanism-related cause $(N=47)$. Table 2 presents the results, including unstandardized and standardized beta coefficients and percentage of variance explained in NDI by each model across the six conditions. 
Using the entire sample, a model that included pain intensity, PCS, HADS ${ }_{\text {dep }}$, CROM, and UFT PPT was able to explain $69.2 \%$ of variance in NDI score, with pain intensity accounting for the greatest variance. This result was repeated for the posture and movement subgroup, with the difference being the replacement of the $\mathrm{HADS}_{\text {dep }}$ with the HADS $\mathrm{anx}$ in the final model. The model for this subgroup explained $65.5 \%$ of total NDI variance.

The results of the emotional group are notably different. The HADS ${ }_{\text {dep }}$ score explained the greatest variance in NDI score in this group, followed by CROM. Pain intensity explained only $5.8 \%$ unique variance in NDI. This was one of only two groups in which TA PPT explained significant unique variance in NDI score while UFT PPT was not retained. This was also one of only two groups for which PCS was not retained. The model for this group was the most robust, explaining $86.6 \%$ of overall variance in NDI score.

In those subjects who described the cause of their symptoms in terms of a predisposition or lifestyle factor, a model including pain intensity, PCS, and UFT PPT explained $61.9 \%$ of variance in NDI. The PCS was the greatest predictor of NDI in this group. This was one of only two groups in which the HADS was not retained.

The model for the group that described at least one mechanistic or structural cause for their symptoms contained only two predictors: pain intensity and PCS, explaining $61.5 \%$ of variance in that group's NDI score. Similar to the first two groups, pain intensity explained the greatest variance here.

\section{Discussion}

The purpose of this study was to explore the causes of neck pain as reported by people who experience the condition. A secondary purpose was to determine how neckrelated disability could be explained when the database was stratified by causal theme. Using a large database and a robust descriptive methodology, we identified 8 causal themes from 321 reported causes. These themes ranged from purely biological (structural and mechanistic causes) to psychological (emotional causes) and social/environmental (interventional causes, environmental causes) supporting a biopsychosocial understanding of neck-related disability. A series of exploratory regression analyses subsequently suggested that disability can be explained by different factors when data are stratified by the patients' perception of the cause of their neck pain.

Current models of patient-centred care encourage exploration of the patient's understanding of their illness and treatment options $[34,35]$. It appears that people with neck pain may think about the causes of their symptoms in different ways, in keeping with the central theories of the illness representations model. Of notable interest here is that different causal beliefs may be associated with the resultant disability experience. The superficial clinical application would be to encourage clinicians to explore their patients' perceptions of neck pain, but caution with such generalization must be exercised. Despite a large (by qualitative standards), heterogenous, and representative sample with theoretically reasonable themes, qualitative approaches to research should not be considered inherently generalizable. This study represents more of a hypothesis generation rather than hypothesis testing exercise. While valuable, potentially offering new pathways to neck pain research, assumptions of generalizability should be tempered until these findings have been confirmed in other samples and through more objective methodologies.

Previous qualitative research using general physicians as informants has suggested that patients with neck pain have difficulty accepting psychological explanations for their symptoms [36]. Based on the results of our study, it would appear that a more accurate statement would be that some patients have difficulty accepting a psychological explanation, as $30 \%$ of the subjects in our study volunteered a psychological explanation. Of notable interest was that those who recognized a psychological or emotional trigger appeared to have a disability experience that was driven primarily by negative affect, in this case, score on the HADS depression subscale. This is in contrast to the majority of recently published models explaining neck-related disability, which tend to suggest that pain intensity and catastrophic beliefs are the primary cross-sectional predictors of neckrelated disability [8,37-39]. This particular subgroup was also notable for the retention of PPT at an anatomically distal site (tibialis anterior) in the final model. It has been suggested the reduced PPT at a distal site is indicative of dysfunction in the central nociceptive processing pathways, more commonly seen in those with neck pain of traumatic etiology [40]. Recent evidence has also emerged to suggest that pain threshold, most notably thermal pain, has an association with psychological states including depression $[41,42]$. This raises the potential for a shared mechanism between depressive mood, pain, and nociceptive processing, and a potential mechanistic pathway for our findings.

The subgroup that indicated that their neck symptoms were caused by some predisposition or lifestyle behaviour, such as genetics, arthritis, or a general lack of fitness, also deviated from the traditional model of disability. The regression model in this group suggested that pain catastrophizing accounted for considerably greater variance in disability $(41.8 \%)$ than did pain intensity (12.7\%). This novel finding is difficult to interpret. It is possible these people accept neck pain as a normal part of their daily experience. Perhaps those subjects are more accustomed to experiencing low level neck pain and do not feel overly disabled by the experience. Perhaps they expect to "just live with it." If this were the case, then another variable would account for greater variance in disability, and of those that we collected, pain catastrophizing contributed more strongly. This is an interesting field for future study.

Readers will note that we have limited the number of cross-group statistical comparisons. For example, we have not compared mean pain, disability, or catastrophizing between the subgroups. There are two reasons for this, the first being that, owing to the exploratory nature of the primary study purpose, it would have been impossible to calculate and justify a sample size for conducting those 
comparisons, and such post-hoc analyses risk spurious findings. However, a stronger reason is that the subgroups were not unique, insofar as some subjects would have been assigned to 2 or even 3 subgroups. This violates a key assumption of group comparisons being that observations between groups are independent and therefore free of systematic bias. Since we could not have satisfied this assumption, such comparisons would have been inappropriate.

There are key limitations to this exploratory study that must be observed. The regression models, while meeting the basic statistical assumptions, may not be robust given the sample sizes in each theme. In the smaller themes (i.e., emotional and predisposition/lifestyle) it was difficult to confidently declare homoscedasticity, and as such the models may not perform equally well across the range of NDI scores. We are also unable to determine whether subjects provided responses that related to the initiation of their neck symptoms, or to the maintenance or exacerbation of their neck symptoms. This is arguably irrelevant in that the perspective of the patient is what matters here, rather than their "correctness." Nonetheless, interpretation of the results should consider the nature of the question and responses. Finally, readers should consider the subjects and the sampling frame. All subjects for this analysis were sampled from a primary care clinical setting. While we do not anticipate this to have a dramatic effect on the themes themselves, we do expect that the relative proportions of responses within each theme would likely be different had the sampling frame been the general community. As an example, we suspect that people with traumatic causes of neck pain are overrepresented in the clinical setting as compared to the community. Readers should be aware of the sampling frame when interpreting our results.

In summary, we have identified 8 themes that explain the different ways people with neck pain think about the cause(s) of their symptoms. The larger themes (posture and movement, emotions, predisposition and Lifestyle, structure and mechanism) were explored more deeply to find that the experience of disability may be predicted in different ways across the themes. Clinicians are encouraged to explore these perceptions and to consider the results of this study when making decisions on the most appropriate intervention strategy. For example, a patient who understands the causes of their symptoms in primarily postural or movementrelated terms may benefit most from pain-reducing interventions. Those who perceive primarily emotional causes may benefit more from formal psychotherapy to address issues of depression if present, while those who believe a predisposition or lifestyle behaviour is the cause of their symptoms may benefit from more cognitively-oriented interventions aimed at catastrophic thinking. This is purely speculation at this point, but supports a new pathway towards evaluating treatment for neck pain that does not consider all mechanical neck pain as homogenous. These findings fit nicely within, and offer options for expansion of, the neck pain framework proposed by the Neck Pain Task Force [3]. That model proposed that the experience of neck pain and disability, and treatment seeking behavior, is influenced by the perception of interference with normal life and a host of "factors" that include psychological, social, and health behaviors. The illness representations model is also useful for understanding the motivations behind different health behaviours, and our results suggest that the perception of interference may be associated with the way the sufferer conceptualizes the causes of their neck symptoms.

\section{References}

[1] P. Côté, V. Kristman, M. Vidmar et al., "The prevalence and incidence of work absenteeism involving neck pain: a cohort of Ontario lost-time claimants," Spine, vol. 33, supplement 4, pp. S192-S198, 2008.

[2] P. R. Croft, M. Lewis, A. C. Papageorgiou et al., "Risk factors for neck pain: a longitudinal study in the general population," Pain, vol. 93, no. 3, pp. 317-325, 2001.

[3] J. Guzman, E. L. Hurwitz, L. J. Carroll et al., "A new conceptual model of neck pain: linking onset, course, and care: the Bone and Joint Decade 2000-2010 Task Force on Neck Pain and Its Associated Disorders," Spine, vol. 33, supplement 4, pp. S14S23, 2008.

[4] B. R. Kaale, J. Krakenes, G. Albrektsen, and K. Wester, "Whiplash-associated disorders impairment rating: neck disability index score according to severity of MRI findings of ligaments and membranes in the upper cervical spine," Journal of Neurotrauma, vol. 22, no. 4, pp. 466-475, 2005.

[5] L. Barnsley, S. M. Lord, B. J. Wallis, N. Bogduk, and M. J. Bolesta, "The prevalence of chronic cervical zygapophysial joint pain after whiplash," Spine, vol. 20, no. 1, pp. 20-26, 1995.

[6] M. J. L. Sullivan, E. Hall, R. Bartolacci, M. E. Sullivan, and H. Adams, "Perceived cognitive deficits, emotional distress and disability following whiplash injury," Pain Research and Management, vol. 7, no. 3, pp. 120-126, 2002.

[7] L. J. Carroll, J. D. Cassidy, and P. Côté, "The role of pain coping strategies in prognosis after whiplash injury: passive coping predicts slowed recovery," Pain, vol. 124, no. 1-2, pp. 18-26, 2006.

[8] M. A. Schmitt, N. L. Van Meeteren, A. De Wijer, F. R. Van Genderen, Y. D. Van Graaf, and P. J. Helders, "Patients with chronic whiplash-associated disorders. Relationship between clinical and psychological factors and functional health status," American Journal of Physical Medicine and Rehabilitation, vol. 88, no. 3, pp. 231-238, 2009.

[9] J. D. Cassidy, J. C. Linda, P. Coté, M. Lemstra, A. Berglund, and Å. Nygren, "Effect of eliminating compensation for pain and suffering on the outcome of insurance claims for whiplash injury," New England Journal of Medicine, vol. 342, no. 16, pp. 1179-1186, 2000.

[10] L. C. Swartzman, R. W. Teasell, A. P. Shapiro, and A. J. McDermid, "The effect of litigation status on adjustment to whiplash injury," Spine, vol. 21, no. 1, pp. 53-58, 1996.

[11] J. M. Romano, J. A. Turner, M. P. Jensen et al., "Chronic pain patient-spouse behavioral interactions predict patient disability," Pain, vol. 63, no. 3, pp. 353-360, 1995.

[12] M. Nordin, E. J. Carragee, S. Hogg-Johnson et al., "Assessment of neck pain and its associated disorders: results of the Bone and Joint Decade 2000-2010 Task Force on Neck Pain and its Associated Disorders," Spine, vol. 33, supplement 4, pp. S101S122, 2008.

[13] E. Blozik, D. Laptinskaya, C. Herrmann-Lingen et al., "Depression and anxiety as major determinants of neck pain: a 
cross-sectional study in general practice," BMC Musculoskeletal Disorders, vol. 10, article 13, 2009.

[14] M. J. L. Sullivan, P. Thibault, M. J. Simmonds, M. Milioto, A. P. Cantin, and A. M. Velly, "Pain, perceived injustice and the persistence of post-traumatic stress symptoms during the course of rehabilitation for whiplash injuries," Pain, vol. 145, no. 3, pp. 325-331, 2009.

[15] H. Leventhal, Y. Benyamini, and S. Brownlee, "Illness representations: theoretical foundations," in Perceptions of Health and Illness, K. J. Petrie and J. A. Weinman, Eds., pp. 19-45, Harwood Academic, Amsterdam, The Netherlands, 1997.

[16] L. A. Phillips, H. Leventhal, and E. A. Leventhal, "Physicians' communication of the common-sense self-regulation model results in greater reported adherence than physicians' use of interpersonal skills," British Journal of Health Psychology, vol. 17, no. 2, pp. 244-257, 2012.

[17] E. Broadbent, K. J. Petrie, J. Main, and J. Weinman, "The brief illness perception questionnaire," Journal of Psychosomatic Research, vol. 60, no. 6, pp. 631-637, 2006.

[18] J. Weinman, K. J. Petrie, R. Moss-Morris, and R. Horne, "The illness perception questionnaire: a new method for assessing the cognitive representation of illness," Psychology and Health, vol. 11, no. 3, pp. 431-445, 1996.

[19] H. Vernon and S. Mior, "The neck disability index: a study of reliability and validity," Journal of Manipulative and Physiological Therapeutics, vol. 14, no. 7, pp. 409-415, 1991.

[20] M. J. L. Sullivan, S. R. Bishop, and J. Pivik, "The Pain Catastrophizing Scale: development and validation,” Psychological Assessment, vol. 7, no. 4, pp. 524-532, 1995.

[21] A. S. Zigmond and R. P. Snaith, "The hospital anxiety and depression scale," Acta Psychiatrica Scandinavica, vol. 67, no. 6, pp. 361-370, 1983.

[22] J. W. S. Vlaeyen, A. M. J. Kole-Snijders, R. G. B. Boeren, and H. Van Eek, "Fear of movement/(re)injury in chronic low back pain and its relation to behavioral performance," Pain, vol. 62, no. 3, pp. 363-372, 1995.

[23] G. F. Spadoni, P. W. Stratford, P. E. Solomon, and L. R. Wishart, "The evaluation of change in pain intensity: a comparison of the $\mathrm{P} 4$ and single-item numeric pain rating scales," Journal of Orthopaedic and Sports Physical Therapy, vol. 34, no. 4, pp. 187-193, 2004.

[24] H. Vernon, "The neck disability index: state-of-the-art, 19912008," Journal of Manipulative and Physiological Therapeutics, vol. 31, no. 7, pp. 491-502, 2008.

[25] J. C. MacDermid, D. M. Walton, S. Avery et al., "Measurement properties of the neck disability index: a systematic review," Journal of Orthopaedic and Sports Physical Therapy, vol. 39, no. 5, pp. 400-417, 2009.

[26] D. Walton and J. Elliott, "A higher-order analysis supports use of the tampa scale of kinesiophobia 11-item version in people with neck pain," Physical Therapy. In press.

[27] D. M. Walton, T. H. Wideman, and M. J. Sullivan, "A Rasch analysis of the Pain Catastrophizing Scale supports its use an interval-level measure," The Clinical Journal of Pain. In press.

[28] G. Clementz, B. Borsbo, and C. Norrbrink, "Burnout in patients with chronic whiplash-associated disorders," International Journal of Rehabilitation Research. In press.

[29] E. P. Cobo, M. E. P. Mesquida, E. P. Fanegas et al., "What factors have influence on persistence of neck pain after a whiplash?" Spine, vol. 35, no. 9, pp. E338-E343, 2010.

[30] C. H. Dominick, F. M. Blyth, and M. K. Nicholas, "Unpacking the burden: understanding the relationships between chronic pain and comorbidity in the general population," Pain, vol. 153, no. 2, pp. 293-304, 2012.
[31] A. D. Wasan, N. K. Anderson, and D. B. Giddon, "Differences in pain, psychological symptoms, and gender distribution among patients with left- vs right-sided chronic spinal pain," Pain Medicine, vol. 11, no. 9, pp. 1373-1380, 2010.

[32] D. M. Walton, J. C. Macdermid, W. Nielson, R. W. Teasell, M. Chiasson, and L. Brown, "Reliability, standard error, and minimum detectable change of clinical pressure pain threshold testing in people with and without acute neck pain," Journal of Orthopaedic \& Sports Physical Therapy, vol. 41, no. 9, pp. 644-650, 2011.

[33] M. Sandelowski, "What's in a name? Qualitative description revisited," Research in Nursing and Health, vol. 33, no. 1, pp. 77-84, 2010.

[34] S. Delon and B. Mackinnon, "Alberta's systems approach to chronic disease management and prevention utilizing the expanded chronic care model," Healthcare Quarterly, vol. 13, pp. 98-104, 2009.

[35] J. H. Robinson, L. C. Callister, J. A. Berry, and K. A. Dearing, "Patient-centered care and adherence: definitions and applications to improve outcomes," Journal of the American Academy of Nurse Practitioners, vol. 20, no. 12, pp. 600-607, 2008.

[36] M. Wermeling, M. Scherer, and W. Himmel, "GPs' experiences of managing non-specific neck pain-a qualitative study," Family Practice, vol. 28, no. 3, pp. 300-306, 2011.

[37] X. Luo, C. L. Edwards, W. Richardson, and L. Hey, "Relationships of clinical, psychologic, and individual factors with the functional status of neck pain patients," Value in Health, vol. 7, no. 1, pp. 61-69, 2004.

[38] R. Nieto, J. Miró, A. Huguet, and C. Saldaña, "Are coping and catastrophising independently related to disability and depression in patients with whiplash associated disorders?" Disability and Rehabilitation, vol. 33, no. 5, pp. 389-398, 2011.

[39] D. P. Thompson, M. Urmston, J. A. Oldham, and S. R. Woby, "The association between cognitive factors, pain and disability in patients with idiopathic chronic neck pain," Disability and Rehabilitation, vol. 32, no. 21, pp. 1758-1767, 2010.

[40] D. Scott, G. Jull, and M. Sterling, "Widespread sensory hypersensitivity is a feature of chronic whiplash-associated disorder but not chronic idiopathic neck pain," Clinical Journal of Pain, vol. 21, no. 2, pp. 175-181, 2005.

[41] L. Piñerua-Shuhaibar, N. Villalobos, N. Delgado, M. A. Rubio, and H. Suarez-Roca, "Enhanced central thermal nociception in mildly depressed nonpatients and transiently sad healthy subjects," Journal of Pain, vol. 12, no. 3, pp. 360-369, 2011.

[42] A. Sjors, B. Larsson, A. L. Persson, and B. Gerdle, "An increased response to experimental muscle pain is related to psychological status in women with chronic non-traumatic neck-shoulder pain," BMC Musculoskeletal Disorders, vol. 12, article 230, 2011. 


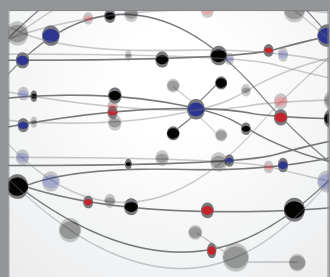

The Scientific World Journal
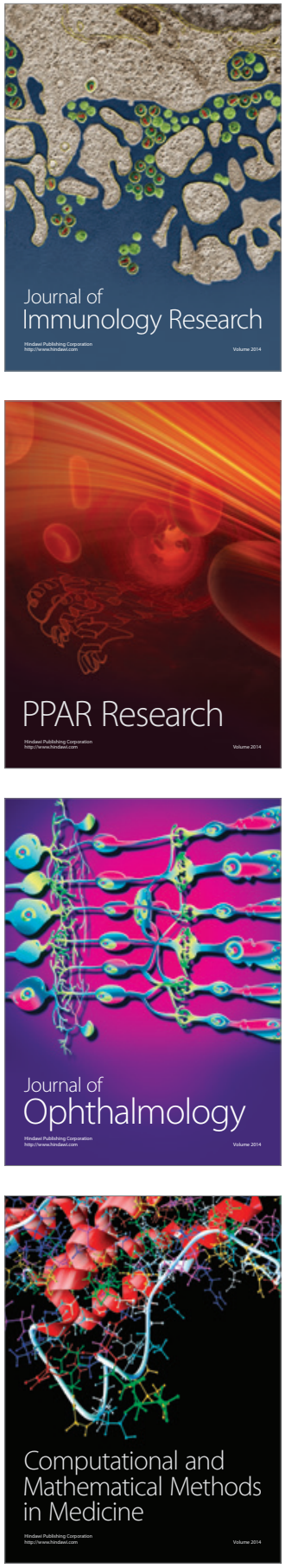

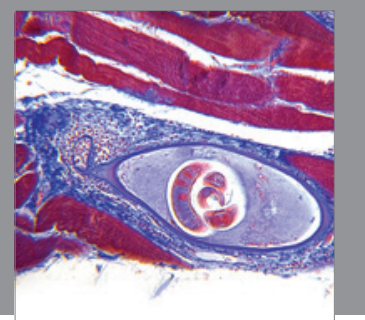

Gastroenterology

Research and Practice
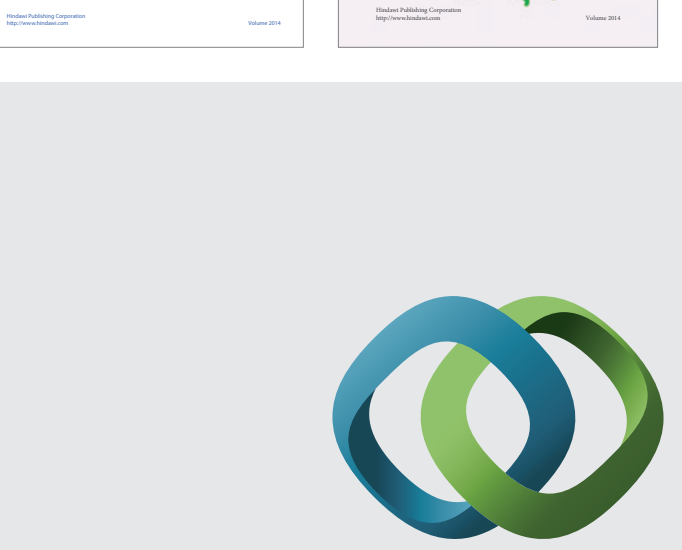

\section{Hindawi}

Submit your manuscripts at

http://www.hindawi.com
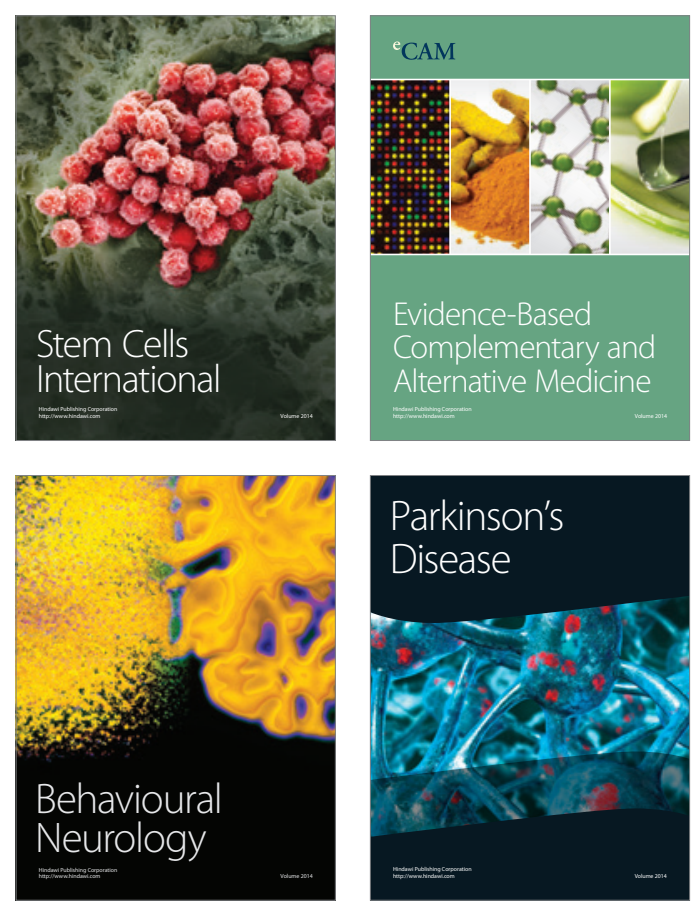

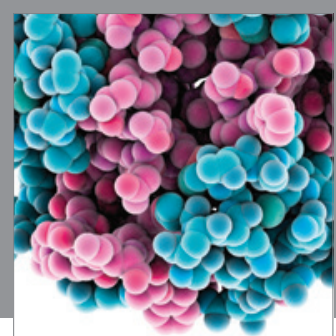

Journal of
Diabetes Research

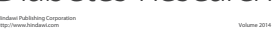

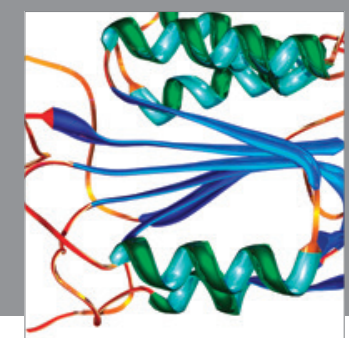

Disease Markers
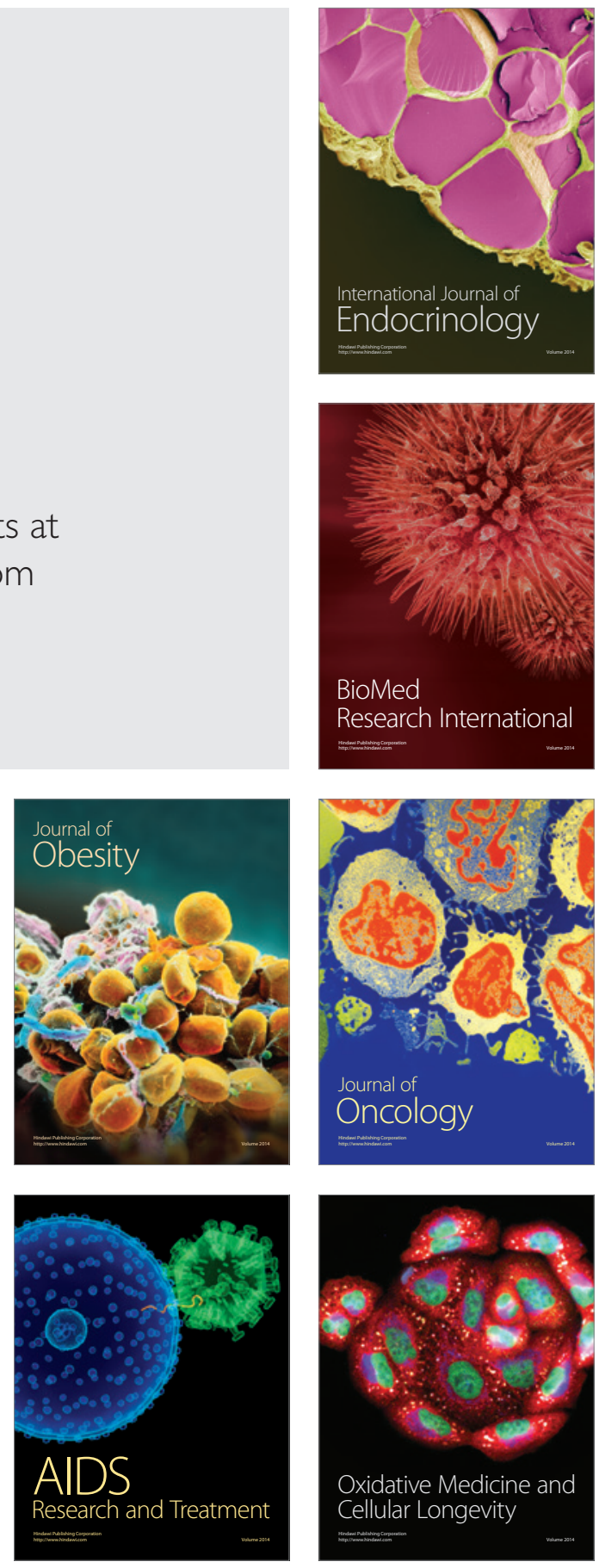